\title{
Magnetic stimulation in the diagnosis of lumbosacral radiculopathy
}

\author{
S CHOKROVERTY, R SACHDEO, J DILULLO, R C DUVOISIN
}

\begin{abstract}
From the Department of Neurology and the Division of Clinical Neurophysiology, UMDNJ-Robert Wood Johnson Medical School and University Hospital, New Brunswick and the Neurology Service, VA Medical Center, Lyons, New Jersey, USA
\end{abstract}

SUMMARY Five patients presenting with sensory-motor disturbances consistent with a clinical diagnosis of L5 or S1 radiculopathy were studied. All had conventional nerve conduction tests and electromyography. The lumbosacral roots were stimulated in the lumbosacral region by using the Cadwell MES-10 Magneto-electric stimulator. The compound muscle action potentials were recorded bilaterally by surface electrodes applied to the soleus and tibialis anterior muscles. The latencies to the affected muscles were significantly prolonged. The appropriate root dysfunction was confirmed at operation or by the imaging techniques. It was concluded that surface stimulation of the lumbosacral roots by a magnetic coil is a potentially useful technique for the non-invasive evaluation of the function of the lumbosacral roots.

A very common problem is low back pain caused by lumbosacral radiculopathy due to lumbar disc disease. Laboratory confirmation of the diagnosis is essential for satisfactory treatment. Electrodiagnosis (electromyography and nerve conduction study) has become a standard supplement to imaging techniques before surgical intervention. However, conventional nerve conduction study does not usually help in the diagnosis. Sometimes a prolonged $\mathrm{H}$ reflex latency on one side may suggest S1 radiculopathy. Electromyography may be helpful but requires needle sampling of many muscles in the limbs, including the paraspinal muscles. The procedure is painful and time consuming. Yet even after such extensive testing the diagnosis may remain uncertain in some patients. Recording of compound muscle action potentials (CMAPs) in the tibialis anterior or soleus muscles after stimulatiion of the lumbosacral roots in the lower back, may evaluate motor conduction along L5 and S1 roots. High voltage percutaneous direct electrical stimulation over the lumbosacral spinal column may be used to assess conduction in the cauda equina, ${ }^{12}$ but this procedure produces discomfort to the subjects.

Recently, it has been possible to stimulate the human brain from the scalp with a magnetic coil, ${ }^{3-5}$

Address for reprint requests: Dr S Chokroverty, PO Box 308, Lyons, NJ 07939, USA.

Received 5 July 1988 and in revised form 28 November 1988. Accepted 12 December 1988 and record consistent CMAPs from the hand and the leg muscles. Central motor conduction can be measured by the method. It is also possible to stimulate peripheral motor pathways such as the relatively inaccessible lumbosacral roots by surface stimulation with a magnetic coil. This approach has the great advantage of being relatively painless. We report the usefulness of such a technique in diagnosing lumbosacral radiculopathy.

\section{Clinical material}

Four patients presented with low back pain radiating to the thigh and one had left thigh pain radiating to the left leg. For 20 years patient 1 had suffered from partial complex seizures which had been controlled by diphenylhydantoin and carbamazepine. Patient 3 was diagnosed in the past to have olivopontocerebellar atrophy based on clinical and radiological findings. All patients had standard electrodiagnostic and neuroradiological tests. The diagnostic considerations consisted of left L5 radiculopathy in patient 1 (fig 1) and right L5 radiculopathy in patient 3 due to lumbar disc lesion, right L5 and S1 radiculopathy associated with lumbar spinal stenosis in patient 4 , and left $S 1$ radiculopathy due to left sacral plasmacytoma in patient 2 . As seen in table 1 the diagnosis was confirmed at operation or by biopsy. In patient 5 left lumbosacral plexopathy was diagnosed for which no cause was found.

\section{Method}

1. Conventional electrodiagnostic study. All patients had conventional electrodiagnostic tests consisting of EMG and 


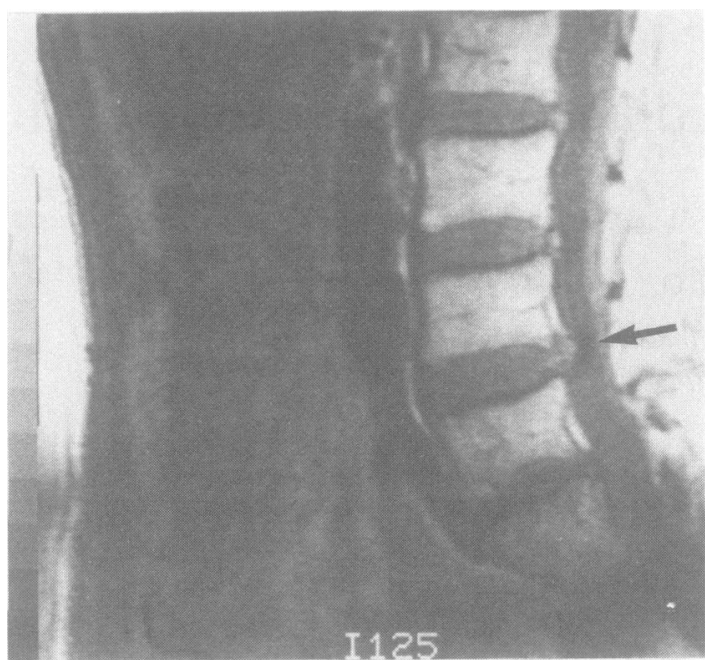

Fig 1 Magnetic resonance imaging of the lumbosacral spine in patient 1 showing a bulging disc (arrow) at L4-L5 level.

nerve conduction study using the standard techniques. ${ }^{6}$ EMG included lumbar paraspinal, quadriceps, biceps femoris, tibialis anterior and medial hamstring muscles. Nerve conduction studies were performed on the peroneal, tibial and sural nerves using Cadwell conventional electrical stimulator (CES). The surface temperature of the legs was kept between $32^{\circ}$ and $34^{\circ} \mathrm{C}$.

2. Magneto-electrical stimulation. Stimulation over the vertebral column was obtained using a Cadwell Magneto-electric stimulator (MES) to stimulate the lumbosacral roots. Potentials were recorded on a Cadwell 5200 A neurodiagnostic system. The subjects were lying down comfortably in the prone position. The magnetic coil was placed in a plane parallel to the lumbosacral spine. The centre of the coil rested over the spinal column precisely in the midline. We then moved the coil vertically from lumbar to sacral region and laterally to obtain responses from midline and lateral placement of the magnetic coil. Surface electrodes were used to record CMAPs from the soleus and tibialis anterior muscles bilaterally. The distances between the distal inner edge of the coil and the recording electrodes were the same on both sides. The reference electrodes were placed on the Achilles tendon or $5-6 \mathrm{~cm}$ below the active electrode on the tibialis muscles. We placed the ground electrode between the stimulating magnetic coil and the recording electrodes. The skin temperature was kept at $32^{\circ}-34^{\circ} \mathrm{C}$. We used stimulus strength of $100 \%$ to obtain maximum amplitude and consistent CMAPs. The latencies and amplitudes were measured bilaterally by cursors. We kept the coil consistently in one orientation while gradually moving it up or down until a consistent and maximal response was obtained. The respon ses were reproducible on repeated stimulation while the coif

Table 1 Pertinent clinical, radiological and operative data in 5 patients with lumbosacral lesion

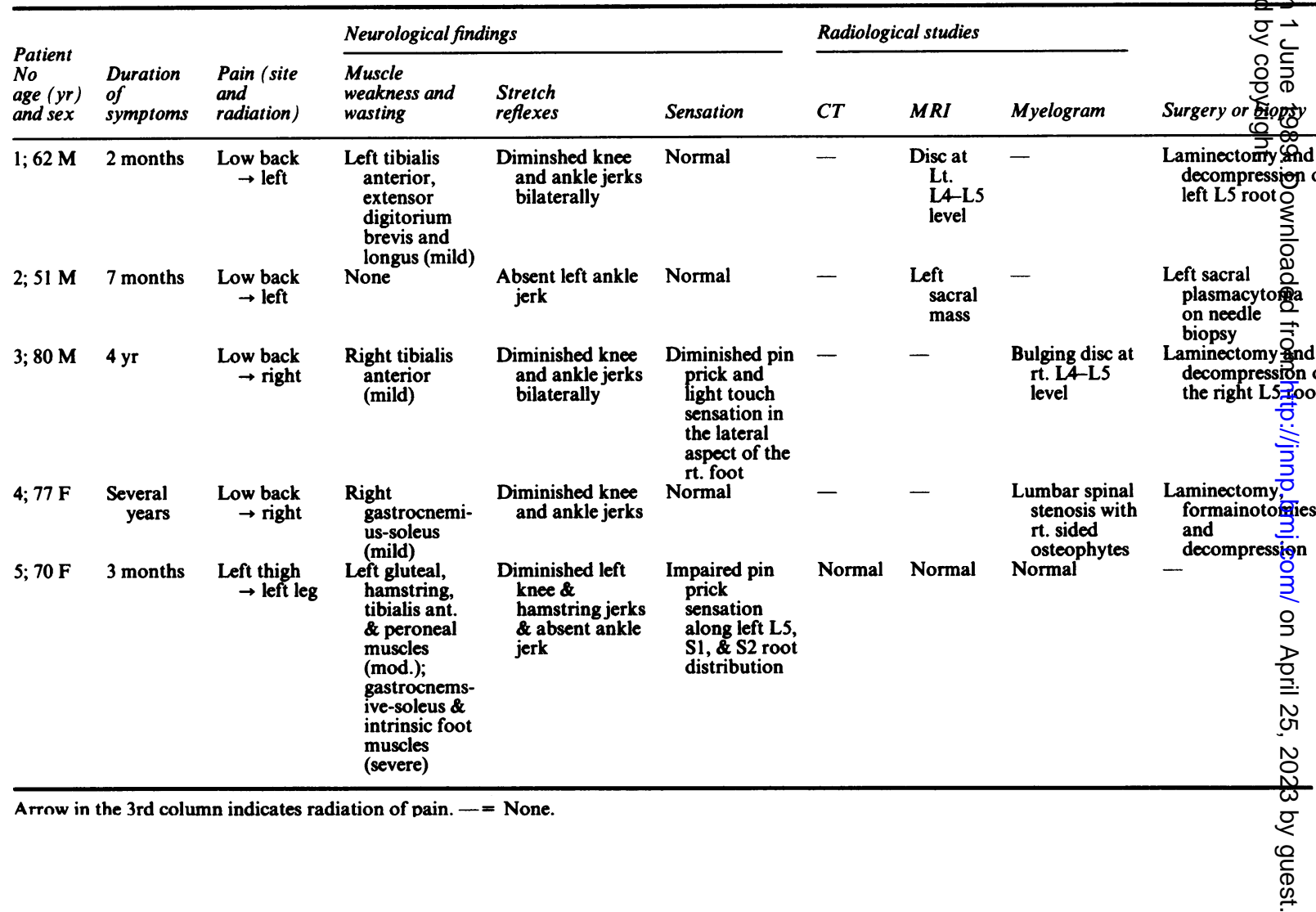


was kept in the same location. The tracings were collected simultaneously with the same stimulus in each patient. The same magneto-electrical stimulation technique was used in seven control subjects free of neuromuscular disease by history and physical examination.

\section{Results}

1. Conventional electrodiagnostic tests. In patient 1 the EMG findings consisted of fibrillation potentials, positive sharp waves and a decreased interference pattern in the left tibialis anterior muscle. The remainder of the EMG was normal. The nerve conduction findings were consistent with bilateral mild sensorymotor axonal type of polyneuropathy in the legs (peroneal nerve conduction velocity $=35 \mathrm{~m} / \mathrm{s}$ with CMAP amplitude $=1.4 \mathrm{mV}$; tibial nerve conduction velocity $=38 \mathrm{~m} / \mathrm{s}$ with CMAP amplitude $=6 \mathrm{mV}$; sural nerve sensory amplitude $=3 \cdot 5 \mu \mathrm{V}$ ).

EMG findings in patient 2 consisted of positive sharp waves, occasional fasciculations, fibrillations and a decreased interference pattern in the left gastrocnemius muscle. The nerve conduction findings distally including CMAP amplitudes were normal.

In patient 3, EMG showed evidence of denervation in the legs manifested by fibrillations and positive sharp waves at rest in at least two places in the same muscles. There was reduced interference pattern, a mixture of long and short duration motor and polyphasic potentials in approximately $20 \%$ of the motor units. The nerve conduction findings were consistent with a sensory-motor axonal neuropathy in the legs (peroneal nerve conduction velocity $=31 \mathrm{~m} / \mathrm{s}$ with CMAP amplitude $=0.6 \mathrm{mV}$; no sensory responses on sural nerve stimulation).

In patient 4 the EMG findings comprised fibrillations and positive sharp waves at rest in the right medial gastrocnemius and right biceps femoris muscles. The interference pattern was decreased in these muscles. The rest of the EMG and the nerve conduction studies distally including CMAP amplitudes were normal. These findings were consistent with S1 radiculopathy on the right side.

In patient 5 EMG findings consisted of fibrillation and positive sharp waves at rest and a decreased interference pattern in the left gastrocnemius, left biceps femoris and left gluteus maximus muscles. The remainder of the EMG including the paraspinal EMG was normal. Left sural nerve stimulation showed no sensory potentials and the rest of the nerve conduction findings including CMAP amplitudes were normal. A repeat EMG study 6 weeks later also showed evidence of denervation in the left tibialis anterior and the left paraspinal muscles.

2. Magneto-electrical stimulation. In each individual we were able to obtain consistent CMAPs in the soleus and tibialis anterior muscles. It should be noted that it was not possible to locate precisely the site of lumbosacral roots and, therefore, we moved the coil up and down until we obtained the maximal response from the appropriate muscles. We selected soleus to evaluate $S 1$ root function and tibialis anterior muscle to evaluate L5 root function. We obtained the maximal and reproducible CMAPs at least four times in each individual after placing the magnetic coil over the lumbosacral spinal column. The distance from the L4 spine to the distal inner edge of the coil over the lumbosacral vertebral column varied from $5-12 \mathrm{~cm}$ in different subjects but the placement of the coil was identical in each subject while recording simultaneously from two sides.

We noted a change in the orientation of the coil if for example, it was placed upside down or if the handle of the stimulating coil was rotated to either side. This caused a distinct change in the amplitude, configuration and latency of the CMAP. These changes appear to have resulted from a change in the direction of the magnetic flux and, therefore, a change in the direction

Table 2 Latencies in milliseconds from the distal inner edge of the magnetic coil over the central lumbosacral region to the onset of the -MAPs in the tibialis anterior (TA) and soleus muscles in 5 patients, and the mean (standard deviation) of the latencies in 7 controls. The IMAP amplitudes, distance in $\mathrm{cm}$ from the distal inner edge of the coil to the active recording electrode and the height are given

\begin{tabular}{|c|c|c|c|c|c|c|c|c|c|c|c|c|c|c|c|}
\hline \multirow{2}{*}{$\begin{array}{l}\text { Datients } \\
\text { Vo/Age in } \\
\text { rrs \& sex } \\
\text { \& controls }\end{array}$} & \multicolumn{3}{|c|}{ Latencies to $T A$} & \multicolumn{3}{|c|}{$\begin{array}{l}\text { CMAP amplitude } \\
(M V): T A\end{array}$} & \multirow[b]{2}{*}{$\begin{array}{l}\text { Distance } \\
\text { to TA }\end{array}$} & \multicolumn{3}{|c|}{ Latencies to soleus } & \multicolumn{3}{|c|}{ CMAP ampitude: Sol } & \multirow[b]{2}{*}{$\begin{array}{l}\text { Distance } \\
\text { to sol }\end{array}$} & \multirow[b]{2}{*}{$\begin{array}{l}\text { Height } \\
\text { (inches) }\end{array}$} \\
\hline & $R t$ & $L t$ & $\begin{array}{l}\text { Rt-Lt } \\
\text { Diff }\end{array}$ & $R t$ & $L t$ & $\begin{array}{l}\text { Rt-Lt } \\
\text { Diff }\end{array}$ & & $R t$ & $L t$ & $\begin{array}{l}\text { Rt-Lt } \\
\text { Diff }\end{array}$ & $R t$ & $L t$ & $\begin{array}{l}\text { Rt-Lt } \\
\text { Diff }\end{array}$ & & \\
\hline $\begin{array}{l}; 62 \mathrm{M} \\
: 51 \mathrm{M} \\
; 80 \mathrm{M} \\
; 77 \mathrm{~F} \\
; 770 \mathrm{~F}\end{array}$ & $\begin{array}{l}\frac{17.6}{17.8} \\
14.7 \\
11.2\end{array}$ & $\begin{array}{l}\frac{19 \cdot 1}{15 \cdot 3} \\
12 \cdot 6 \\
16 \cdot 2\end{array}$ & $\begin{array}{l}1 \cdot 5 \\
\frac{7}{2 \cdot 5} \\
2 \cdot 1 \\
5 \cdot 0\end{array}$ & $\begin{array}{l}\frac{1.2}{-} \\
1.5 \\
1.6 \\
2.9\end{array}$ & $\begin{array}{l}1 \cdot 4 \\
2 \cdot 1 \\
2 \cdot 1 \\
0 \cdot 1\end{array}$ & $\begin{array}{l}0.2 \\
0.6 \\
0.5 \\
2.8\end{array}$ & $\begin{array}{l}\frac{60 \cdot 5}{\overline{66}} \\
55 \\
61\end{array}$ & $\begin{array}{l}21 \cdot 0 \\
14 \cdot 5 \\
17.4 \\
15 \cdot 1 \\
12 \cdot 8\end{array}$ & $\begin{array}{l}21.0 \\
19.9 \\
17.4 \\
12.8 \\
21.2\end{array}$ & $\begin{array}{l}0 \\
5 \cdot 4 \\
0 \\
2 \cdot 3 \\
8 \cdot 4\end{array}$ & $\begin{array}{l}2.4 \\
2.1 \\
3.0 \\
0.6 \\
5.5\end{array}$ & $\begin{array}{l}2 \cdot 6 \\
0 \cdot 3 \\
4 \cdot 4 \\
2 \cdot 7 \\
0 \cdot 1\end{array}$ & $\begin{array}{l}0 \cdot 2 \\
1.8 \\
1.4 \\
2 \cdot 1 \\
5 \cdot 4\end{array}$ & $\begin{array}{l}63 \\
61 \\
67 \\
53 \\
62\end{array}$ & $\begin{array}{l}72 \\
72 \\
76 \\
68 \\
66\end{array}$ \\
\hline $\begin{array}{l}\text { Zontrols } \\
\text { Mean age } \\
=58 \text { ) }\end{array}$ & $\begin{array}{l}12 \cdot 1 \\
(1 \cdot 2)\end{array}$ & $\begin{array}{l}12 \cdot 4 \\
(1 \cdot 5)\end{array}$ & $\begin{array}{l}0 \cdot 3 \\
(0 \cdot 3)\end{array}$ & $\begin{array}{l}2.0 \\
(0.9)\end{array}$ & $\begin{array}{l}1.9 \\
(0.9)\end{array}$ & $\begin{array}{l}0 \cdot 3 \\
(0 \cdot 2)\end{array}$ & $\begin{array}{l}62 \cdot 2 \\
(4 \cdot 2)\end{array}$ & $\begin{array}{l}12 \cdot 8 \\
(1 \cdot 1)\end{array}$ & $\begin{array}{l}13 \cdot 0 \\
(1 \cdot 3)\end{array}$ & $\begin{array}{l}0 \cdot 2 \\
(0 \cdot 3)\end{array}$ & $\begin{array}{l}7.9 \\
(3.4)\end{array}$ & $\begin{array}{l}8 \cdot 9 \\
(4 \cdot 6)\end{array}$ & $\begin{array}{l}2 \cdot 6 \\
(2 \cdot 7)\end{array}$ & $\begin{array}{l}61 \cdot 5 \\
(5 \cdot 9)\end{array}$ & $\begin{array}{l}65 \cdot 5 \\
(3 \cdot 0)\end{array}$ \\
\hline
\end{tabular}

-: Not measured. 
of the induced current. Another important observation was that on moving the coil $2-6 \mathrm{~cm}$ to either side of the spine there was a paradoxical response. On lateral stimulation the amplitude of the ipsilateral CMAP was considerably attenuated while the contralateral CMAP remained unchanged. There was also a change in the latency of the CMAP but we did not measure the latencies on lateral stimulation.

We noted a linear relationship between the strength of stimulation and the amplitude of the evoked CMAP. As the strength of stimulation was increased the amplitude was also increased until it became maximal and consistent.

Table 2 lists the latencies in milliseconds from the distal inner edge of the coil over the central lumbosacral region to the onset of the CMAPs in five patients as well as the mean and standard deviation of the latency in seven controls. A difference in latency of more than 1 millisecond between two sides was considered abnormal. This value is more than 2.5 standard deviation above the mean (table 2). As seen in table 2 the latencies to soleus and tibialis anterior muscles were bilaterally prolonged in patients 1 and 3 as compared with our controls. Conventional electrical studies in these two patients showed evidence of polyneuropathy in the lower limbs thus accounting for the bilaterally prolonged latencies after MES in these patients. Long-term diphenylhydantoin therapy in patient 1 and olivopontocerebellar atrophy in patient

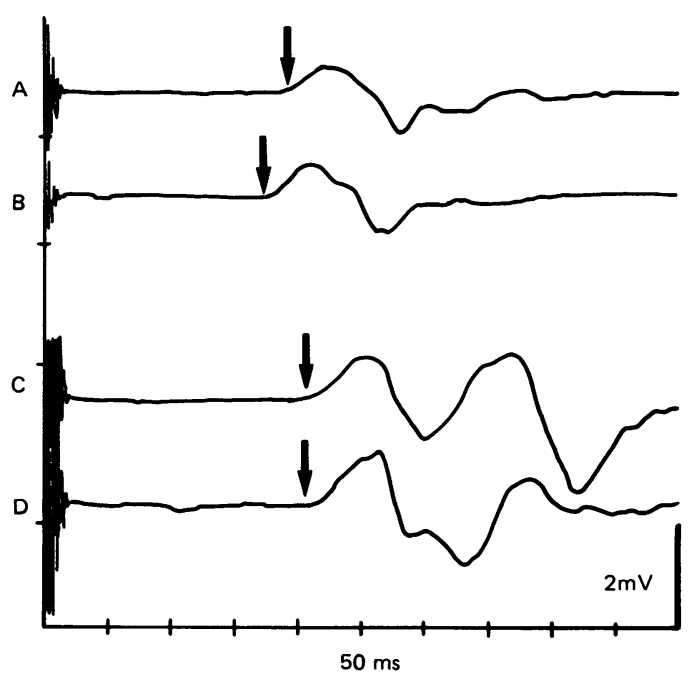

Fig 2 Magneto-electric stimulation (MES) of the central lumbosacral region in patient 1. Compound muscle action potentials (CMAPs) obtained from the: A. Left tibialis anterior B. Right tibialis anterior C. Left soleus D. Right soleus muscles. Site of stimulation: central lumbosacral region. Note prolonged CMAP in left tibialis anterior muscle.
3 were causally related to polyneuropathy in these two patients. However, when one compares the latencies to the affected muscles with those obtained from the unaffected muscles, it is clear that there was a sig-

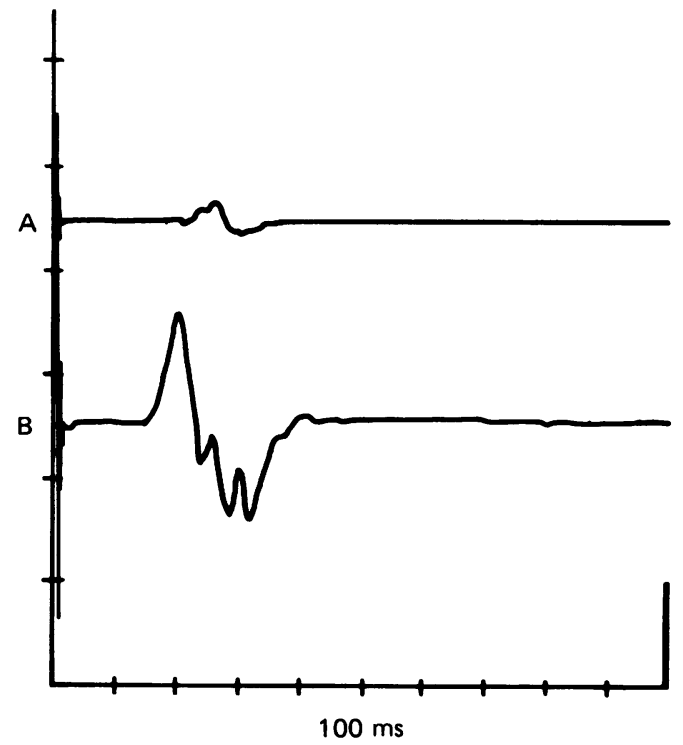

Fig 3 CMAPs after MES stimulation of the lumbosacral region in patient 2 . A. left soleus $B$. right soleus muscles. Note prolonged latency and reduced CMAP in the left soleus.

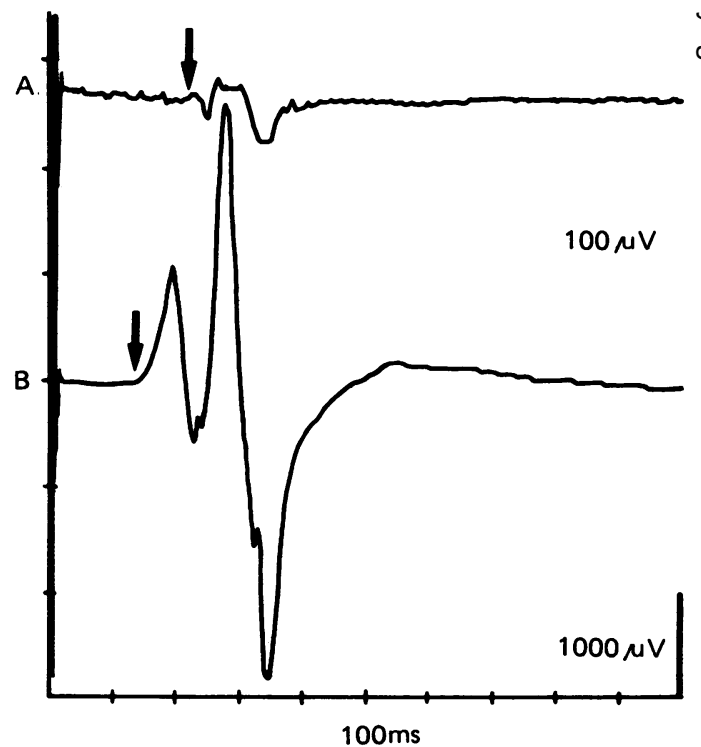

Fig 4 CMAPs after MES stimulation in patient 5. A. left soleus $B$. right soleus muscle. Note marked reduction of the CMAP amplitude in the left and prolongation of latency to the same muscle. 
nificant prolongation to the affected tibialis anterior muscles. There was also a significant difference in latencies between the affected and unaffected sides in both these patients after lumbosacral root stimulation indicating affection of L5 root on the left side in patient 1 and on the right side in patient 3 . In the remaining patients there was also a marked difference in the latencies between the affected and unaffected sides. The most marked prolongation in the latency on the affected side was noted in patient 5 . In addition, the amplitude of the CMAP was smaller in the affected than in the unaffected muscles particularly in patients 2 and 5 (table 2). Figures $2-4$ represent samples from patients 1,2 and 5 .

The values in table 1 indicate dysfunction of the left L5 root in patient 1 , the left $S 1$ root in patient 2 , the right $L 5$ root in patient 3 , the right $L 5$ and $S 1$ roots in patient 4 and the left $\mathrm{L} 5$ and $\mathrm{S} 1$ roots in patient 5.

\section{Discussion}

The usefulness of magnetic stimulation in the diagnosis of dysfunction of L5 and S1 roots is shown in these five patients. The diagnosis was confirmed by other methods, radiological studies (cases 1-4), biopsy (case 2) or by operative findings (cases 1, 3 and 4). Whether MES is superior to other procedures in its ability to diagnose root lesion at an early stage when other techniques failed remains to be determined. If our findings are confirmed in a large number of patients with radiculopathy or plexopathy then at least we have a new, non-invasive, simple and painless technique to diagnose such lesions.

Noordhout et al ${ }^{1}$ were able to stimulate lumbosacral roots in humans by percutaneous high voltage electrical stimulator over the lumbosacral spinal column. By using a magnetic coil stimulator we were able to produce consistent CMAP from L5 and S1 innervated muscles after surface stimulation of the lumbosacral roots over the lumbosacral vertebral column. This stimulation is relatively painless in contrast to the discomfort produced by high voltage, percutaneous, electrical stimulation over the lumbosacral spinal column. ${ }^{2}$

One of the problems encountered was the lack of precision on the exact point of stimulation. It is thought that the induced current generated by the magnetic flux in the Cadwell coil is maximal in the region of the circumference of the coil from the inner edge to $2 \mathrm{~cm}$ toward the centre from the inner edge. ${ }^{7}$ Our previous data agree with this conclusion, ${ }^{8}$ while Evans ${ }^{9}$ could not locate the precise point of stimulation. We therefore measured the distance from the distal inner edge of the coil in the lumbosacral region to the active recording electrode on the appropriate muscle.
The difficulty of locating from the surface the exact site of stimulations of the L5 and S1 roots and the lack of precision regarding the maximum site of stimulation are disadvantages of MES. We measured latency rather than conduction time or conduction velocity. Measurement of conduction velocity requires stimulation at two separate points in the nerves or the roots and stimulation of the fast conducting fibres transmitting the impulses along the same nerve fibres to the appropriate muscles. We were unable to locate the roots with sufficient precision and measure the distances accurately between the sites of stimulation and the sites of recordings in the appropriate muscles to permit calculation of conduction velocities. For these reasons measurement of the latency was more appropriate than the conduction velocity. The muscle responses, however, should be approximately similar in configuration, amplitude and latency between the two sides at the same diistance in normal individuals. Whether we obtained supramaximal CMAPs in the controls and the unaffected sides of the patients cannot be determined. As discussed in the Results the CMAP amplitudes in patients 2,4 and 5 were normal after peripheral nerve stimulation. Therefore a considerable reduction of CMAP amplitude after proximal stimulation of the lumbosacral roots may suggest conduction block at a proximal level. However, uncertainty about obtaining supramaximal CMAP consistently after lumbosacral root stimulation makes this a provisional suggestion.

We found that the magnetic coil must be positioned in the midline otherwise the CMAPs will differ in amplitude, configuration and latencies. Displacing the coil laterally by $2-6 \mathrm{~cm}$ produced a paradoxical response, that is, the response was normal in amplitude contralaterally but was considerably attenuated ipsilaterally. ${ }^{10}$ The reasons offered by Cadwell ${ }^{11}$ for this effect are as follows: no current flows through the centre of the coil. When the coil is moved to the right, then the centre of the coil is over the right lumbosacral roots (for example, L5 or S1 roots) and the circumference of the coil is still over the left roots. The induced current is thus parallel to the left roots and effective. Similarly when the coil is moved to the left, the centre of the coil lies over the left roots and the edge of the coil is over the right roots. The induced current is now parallel to the roots on the right side and the current flows along the circumference over the right sided roots.

Despite imprecision on the exact point and site of stimulation our conclusions (based on this limited study) are that surface stimulation of the lumbosacral roots by a magnetic coil is a potentially useful technique for the non-invasive evaluation of the functions of the lumbosacral roots. It has great potential as a simple, relatively painless procedure for 
diagnosis of lumbosacral radiculopathy or plexopathy.

\section{References}

1 Noordhout AM, Rothwell JC, Thompson PD, Day BL, Marsden CD. Percutaneous electrical stimulation of lumbosacral roots in man. J Neurol Neurosurg Psychiatry 1988;51:174-81.

2 Swash M, Snooks SJ. Slowed motor conduction in lumbosacral roots in cauda equina lesions: A new diagnostic technique. J Neurol Neurosurg Psychiatry 1986;49:808-16.

3 Barker AT, Freeston IL, Jalinous R, Jarratt JA. Magnetic stimulation of the human brain and peripheral nervous system: An introduction and the results of an initial clinical evaluation. Neurosurgery 1987;20: $100-9$.

4 Barker AT, Jalinous R, Freeston IL. Non-invasive magnetic stimulation of the human motor cortex. Lancet 1985;i:1106-7.

5 Mills KR, Murray NMG, Hess CW. Magnetic and electrical transcranial brain stimulation: physiological mechanisms and clinical applications. Neurosurgery
1987;20:164-8.

6 Kimura J. Electrodiagnosis in diseases of nerve and muscle. Philadelphia: F A Davis, 1983.

7 Hallett M, Cohen LG, Nilsson J, Panizza M. Differences between electric and magnetic stimulation of human peripheral nerve and motor cortex. In: Chokroverty $S$, ed. Magnetic Stimulation in Clinical Neurophysiology. Stoneham, MA: Butterworth, 1989 (In press).

8 Chokroverty S. Magnetic stimulation of the human peripheral nerves. Electromyogr Clin Neurophysiol (In press).

9 Evans BA. The differences between electrical and magnetic stimulation in the peripheral nervous system. In: Chokroverty S, ed. Magnetic Stimulation in Clinical Neurophysiology. Stoneham, MA: Butterworth, 1989 (In press).

10 Chokroverty S, Spire JP, DiLullo J, Moody E, Maselli R. Magnetic stimulation of the human peripheral nervous system. In: Chokroverty S, ed. Magnetic Stimulation in Clinical Neurophysiology. Stoneham, MA: Butterworth, 1989 (In press).

$11 \mathrm{Cadwell} \mathrm{J}$. The magnetic stimulator and the principles of magnetism. In: Chokroverty S, ed. Magnetic Stimulation in Clinical Neurophysiology. Stoneham MA: Butterworth, 1989 (In press). 